\title{
Experiencia con metodología activa (Aprendizaje Basado en Problemas, ABP) adaptando su evaluación en estudios de Organización de empresas.
}

\section{Experience with active methodology (Problem Based Learning, $P B L)$ and adapting its evaluation in Business Organization \\ Studies. \\ Leyla Angélica Sandoval Hamón ${ }^{1}$.}

Fecha de recepción: 20/01/2019; Fecha de revisión: 22/02/2019; Fecha de aceptación: 25/03/2019

Cómo citar este artículo:

Sandoval Hamón, L. A. (2019). Experiencia con metodología activa (Aprendizaje Basado en Problemas, ABP) adaptando su evaluación en estudios de Organización de empresas. Revista de Innovación y Buenas Prácticas Docentes, 8, 87-96.

Autor de Correspondencia: angelica.sandoval@uam.es

\section{Resumen:}

El proceso de enseñanza-aprendizaje en un entorno universitario tiene en cuenta metodologías alternativas además de las tradicionales como una forma de preparar a los profesionales frente a los desafíos del futuro. Una de estas metodologías es el aprendizaje basado en problemas (ABP), donde los estudiantes pueden abordar un problema real y proponer una solución. Por lo tanto, este trabajo describe la experiencia de aplicar la metodología ABP en la Organización Empresarial de 2017 a 2018. La implementación de esta experiencia requirió un cambio de todos los elementos específicos del tema (por ejemplo: sistema de contenido, planificación, monitoreo y evaluación). Las conclusiones (derivadas de la observación, análisis y evaluación del uso de la metodología ABP, así como las encuestas oficiales de los estudiantes) indican que este tipo de metodología alienta a los estudiantes a desarrollar nuevas habilidades (pensamiento crítico y práctico, análisis y resolución de problemas, trabajo en equipo, habilidades de comunicación, gestión del tiempo, entre otros) y construir su aprendizaje basado en teorías y problemas de las organizaciones contemporáneas.

Palabras clave: Universidad, Organización, Aprendizaje por Experiencia, Evaluación.

\begin{abstract}
:
The teaching-learning process in a university setting takes into consideration alternative methodologies in addition to traditional ones as a way to prepare professionals facing the challenges of the future. One of these methodologies is problem-based learning $(\mathrm{PBL})$, where students can address a real problem and propose a solution. Thus, this work describes the experiment of applying the PBL methodology on Business Organization from 2017 to 2018 . The implementation of this experiment required a change of all subjectspecific elements (for example: content, planning, monitoring and evaluation system). Conclusions (derived from the observation, analysis and evaluation of the use of the PBL methodology, as well as the official surveys of the students) indicate that this type of methodology encourages students to develop new skills (critical and practical thinking, analysis and resolution of problems, teamwork, communication skills, time management, among others) and build their learning based on theories and problems of contemporary organizations.
\end{abstract}

Key Words: University, Organization, Experiential learning, Evaluation.

\footnotetext{
1 Universidad Autónoma de Madrid (España), correo electrónico: angelica.sandoval@uam.es; CÓDIGO ORCID: https://orcid.org/0000-0003-1213-8990
} 


\section{INTRODUCCIÓN}

"El proceso de Bolonia (1999), con el que se inicia desde las instituciones universitarias la respuesta a los cambios importantes que está experimentando el entorno global de la educación superior en Europa, se sigue desarrollando, en estos momentos como un proceso imparable..."(March, 2006, p. 37). Esta adaptación implica en lo relacionado a la docencia, considerar principalmente la reformulación de los planes curriculares, de las metodologías empleadas y de los sistemas de evaluación. En el caso de las metodologías y de los sistemas de evaluación, se han ido desarrollando alternativas a las tradicionales (clase magistral y pruebas tipo test) para tener la posibilidad de obtener más perspectivas y diferentes formas de participación de los estudiantes.

Los estudios centrados en metodologías activas y evaluación formativa han aumentado considerablemente en los últimos años (Exley, \& Dennick, 2007; FernándezPérez, 1989; López-Noguero, 2007; López-Pastor, Castejón, Sicilia-Camacho, NavarroAdelantado, \& Webb, 2011). El proceso de Bolonia (1999) es una de las principales razones que propició que se prestará un particular interés, aunque lo cierto es que la inconformidad de parte de la comunidad educativa con los modelos tradicionales ha sido otro factor que ha dirigido la atención a alternativas de metodologías docente.

Además, Lemus (2006) indica que la metodología activa se ha convertido en el aprendizaje más interesante e innovador de la educación actual. De hecho, una definición común es "el aprendizaje activo implica proporcionar oportunidades a los estudiantes para hablar y escuchar de manera significativa, escribir, leer, y reflexionar sobre los contenidos, ideas, problemas y preocupaciones de una materia académica"(Meyers, \& Jones, 1993, p. 6).

En este escenario, uno de los debates más fructíferos se ha centrado en la reflexión sobre la renovación metodológica, favoreciendo estas metodologías activas (tales como: aprendizaje basado en problemas, aprendizaje cooperativo, estudios de casos, etc.), otros autores como Zayapragassarazan \& Kumar (2012) identifican algunas estrategias de aprendizaje activo (adicionales a las mencionadas) que han llevado a la mejora de la formación (por ejemplo: mapas conceptuales, redacción colaborativa, Brainstorming, panel de discusión, enseñanza por pares, juego de roles, etc.) y de sistemas de evaluación que, en otras palabras, se traducen en cambios en el rol del profesor y el papel del estudiante como protagonista activo de su aprendizaje dentro de un proceso de evaluación continuo (considerando la evaluación no solo formativa sino compartida).

Así, las metodologías activas permiten obtener aprendizajes profundos y duraderos al lograr la implicación, esfuerzo y trabajo personal de los estudiantes. Estos aprendizajes que superan los básicos (de adquirir y memorizar unos conocimientos) propician y estimulan el desarrollo del pensamiento crítico y aprendizaje autónomo. El activarlos en la docencia da paso a que los estudiantes sean más conscientes de su forma de aprender, lo cual les permite extrapolar sus inquietudes y conocimientos a diversos ámbitos a lo largo de su vida. Esta forma de aprender facilita que los estudiantes exploren nuevas herramientas y accedan con más asiduidad a la sociedad del conocimiento para la construcción personal de su conocimiento.

Una de las metodologías activas que se ha ido empleando más en la docencia son las relacionadas con el aprendizaje basado en problemas (ABP). Algunas de las definiciones que han proliferado de esta metodología son:

"EI ABP es un enfoque de aprendizaje y enseñanza que hace que los estudiantes busquen nuevos conocimientos y habilidades, los ayude a superar preguntas de la vida real y diseñe sus propios estudios y actuaciones" (Baysura, Altun, \& Toy, 2016, p. 15).

"Los estudiantes participan en el aprendizaje de temas auténticos, formulan preguntas, plantean hipótesis, buscan información relevante, planifican una consulta, recopilan datos, piensan críticamente, discuten, comparten ideas, razonan y toman decisiones, desarrollan un producto y lo presentan a un público" (Goldstein, 2016, p. 2). 
"El principio de necesidad de saber / necesidad de hacer también impulsa el proceso de aprendizaje y los inspira a profundizar en los conceptos". (Jacques, Bissey, \& Martin, 2016, p. 36).

Además, las aplicaciones de ABP también contribuyen a equipar a los estudiantes con habilidades del siglo XXI, identificadas como pensamiento crítico, resolución de problemas, creatividad, acceso y reestructuración de la información, uso de recursos digitales, responsabilidad, intercambio de ideas, autocontrol y reconciliación (Bell, 2010).

En lo que se refiere a las estrategias de evaluación se pueden catalogar en dos grandes grupos: las estrategias tradicionales (pruebas de conocimientos abiertas y cerradas, trabajos, proyectos y exámenes orales) a las que se asocia la evaluación sumativa y las estrategias innovadoras (autoevaluación, portafolios, técnicas de observación...) que se asocian a la evaluación formativa.

Asimismo, las tecnologías de la información y las comunicaciones no se pueden dejar aún lado así que, partiendo del uso de Moodle, el cual ayuda al profesorado para crear cursos en línea -asegurando su calidad- y gestionar los resultados del aprendizaje. Este recurso tecnológico ha sido un apoyo en el desarrollo de las metodologías activas "dado que el uso de esta herramienta en las aulas favorece y aumenta el grado de interactividad y colaboración de los estudiantes en sus momentos educativos" (Cavus, Uzunboylu, \& Ibrahim, 2006; Cavus, Uzunboylu, \& Ibrahim, 2007, p. 308).

En definitiva, el objetivo de este trabajo es presentar una experiencia docente donde se buscó contribuir al proceso de construcción del aprendizaje de los estudiantes a través del fomento de las metodologías activas (ABP) y el empleo de las TIC en la asignatura de Organización de empresas en el grado de Administración de Empresas. Además, de realizar una adaptación (teniendo en cuenta las metodologías activas alternativas) en la forma en que se evalúa la asignatura.

\section{DESARROLLO DE LA EXPERIENCIA DE INNOVACIÓN}

\subsection{Contexto}

La experiencia se ha analizado en la asignatura de Organización de empresas (6 créditos) que se imparte en el segundo año del grado de administración y dirección de empresas en la Universidad Autónoma de Madrid. El segundo semestre del curso 20072008 fue el periodo que se analizó en esta experiencia y el número total de estudiantes fue de 29 estudiantes.

En la asignatura Organización de empresas, se pretende que el estudiante conozca las organizaciones actuales y actúe en ellas y que entienda cómo funcionan las organizaciones reales y cómo podemos incidir en su diseño; los docentes han replanteado la forma en la cual imparten la asignatura tomando como referencia la metodología activa el aprendizaje basado en problemas (ABP). Esta metodología "permite a los alumnos realizar investigaciones, integrar teoría y práctica, y aplicar conocimientos y habilidades para desarrollar una solución viable a un problema definido" (Savery, 2015, p. 9). Así, esta alternativa da la posibilidad a los estudiantes de acercarse a las organizaciones reales. 


\subsection{Objetivo}

El análisis de la metodología ABP en la asignatura partía de que los estudiantes lograran el siguiente objetivo: Identificar y proponer una solución para un problema encontrado en una organización real.

El problema debía estar relacionado con algunos de los temas contenidos (ver a continuación) en el programa de la asignatura (que serán la base para la solución propuesta). Todo ello de acuerdo con la metodología Aprendizaje basado en problemas y centrado en el estudiante.

Contenidos de la asignatura:

PRIMERA PARTE: FUNDAMENTOS DE LA TEORÍA DE LA ORGANIZACIÓN

TEMA 1: Los estudios de la organización.

TEMA 2: Evolución de la Teoría de la Organización.

SEGUNDA PARTE: CONCEPTOS Y TEORÍAS BÁSICOS

TEMA 3: La organización y el entorno.

TEMA 4: La estructura social de la organización.

TEMA 5: Organización y tecnología.

TEMA 6: La cultura organizativa.

TEMA 7: La estructura física de las organizaciones.

TEMA 8: Poder, control y conflicto en la organización.

TERCERA PARTE: ASPECTOS PRÁCTICOS DE LA FUNCIÓN

ORGANIZATIVA

TEMA 9: La teoría de la organización en la práctica.

TEMA 10: Nuevos desarrollos de la teoría de la organización.

TEMA 11: Comportamiento organizativo.

\subsection{Indicaciones para el desarrollo de la experiencia}

Indicaciones a los estudiantes: sobre aspectos esenciales, la selección de problema, objetivos de aprendizaje, entregables, criterios de evaluación y cronograma.

a) Aspectos esenciales: debe recogerse información de la organización elegida relevante para la solución del problema planteado. Se valoraba especialmente la realización de entrevistas en soporte vídeo. El trabajo debía recoger una introducción a la organización de la que se trate, un diagnóstico del problema, la información recopilada, las bases teóricas que sustenten la solución propuesta al problema, así como la descripción de la solución. También se valoraban las propuestas concretas de implantación de la solución y los instrumentos de medición y control de la solución implantada. Además, se recogía la bibliografía y documentación recopilada. Los estudiantes debían entregar el trabajo escrito (un informe inicial y uno final), así como presentar ante la clase dicho trabajo

b) Selección del problema:

El problema debe tener un alcance limitado y ser abordable por el equipo (máximo 5 personas).

- El equipo debe ser capaz de obtener el apoyo de los directivos de la organización seleccionada y la información necesaria para plantear una solución profesional al problema.

-El problema deberá poder afrontarse en el plazo establecido.

-El equipo deberá resolver cualquier cuestión relativa a la confidencialidad y propiedad intelectual de los datos obtenidos, así como la autorización pertinente para el uso de los mismos en este contexto académico.

-El problema deberá plantear un reto formativo que resulte interesante para los integrantes del equipo, lo que implica su compromiso con el aprendizaje.

c) Objetivos de aprendizaje:

-Construir la comprensión sistemática de una serie de conocimientos específicos de los Estudios de la Organización. 
- Practicar las habilidades organizativas y directivas derivadas de las correspondientes competencias genéricas y específicas incluidas en la Guía de la asignatura.

- Integrar los conceptos teóricos de la asignatura y transformarlos en herramientas útiles para la solución de problemas en organizaciones reales.

- Fomentar la iniciativa y autonomía de los estudiantes para localizar los problemas y proponer soluciones a los mismos mediante su propia construcción del conocimiento.

-Desarrollar la capacidad para criticar y evaluar el trabajo propio y el ajeno (Evaluación compartida).

-Avanzar en las competencias relativas al trabajo en equipo, la planificación y ejecución de proyectos, así como la ejecución de tareas con disponibilidad limitada de tiempo y de recursos.

d) Evaluación.

Instrumentos de evaluación.

-Contrato para establecer las bases sobre cómo deseo ser evaluado/a en la asignatura de Organización de empresas

-Rubricas

-Entregables

Requisitos mínimos establecidos por el profesor

Los requisitos mínimos del trabajo de ABP toman como punto de referencia las rubricas, entre los aspectos que se consideran están: Contenido presentación, Ordenación información, Habilidades expositivas, Materiales elaborados, Trabajo en equipo, Originalidad, será llegar al menos al aceptable en todos los aspectos que se valoran.

Además, los estudiantes tendrán que considerar los criterios de evaluación de la asignatura en general, es decir:

- Trabajo sobre el problema organizativo (ABP) 55\%

- Presentación de informe inicial y final: $10 \%$

- Evaluación inicial: 5\%

- Evaluación final: 30\%

e) Resumen: Los estudiantes constituirán equipos integrados por cinco personas. Cada equipo debe conseguir el acceso a una organización real (lo que incluye la información relevante para el problema en cuestión), así como el permiso de sus responsables para utilizar dicha información con fines exclusivamente académicos. Una vez acordada con la organización la realización del proyecto, los integrantes del equipo deberán, a partir de la información recopilada (que será especialmente valorada cuando se trate de entrevistas en soporte vídeo) describir la organización, definir el problema, identificar los conceptos teóricos que sustenten una posible solución al mismo, especificar tal solución, proponer un plan de implantación de dicha solución y diseñar los correspondientes instrumentos de control. Es decir, el equipo realizará un proyecto de consultoría a pequeña escala en el que se consideran esenciales la base teórica, la recopilación de información empírica, la calidad y profesionalidad del proyecto, la creatividad (en el diseño de la solución al problema planteado y en la ejecución de las tareas) así como su contribución a la construcción individual y colectiva del conocimiento relativo a los Estudios de la Organización. Los estudiantes deberán elaborar y ampliar su conocimiento a partir de los materiales de estudio proporcionados (preferentemente, a través de Moodle) completándolos con otros identificados por ellos y que resulten pertinentes. 


\subsection{Sugerencias para mejorar el protocolo de desarrollo de esta experiencia}

Entre las cuestiones que se deben tener en cuenta son:

El número máximo de estudiantes en el grupo son 5

Los estudiantes son los que buscan el contacto con la empresa, en caso de que no les sea posible tener acceso a una organización, conviene que el profesor tenga algunas posibilidades a las que puedan optar estos estudiantes. No tienen conocimiento de la metodología de aprendizaje basado en problemas, así que es oportuno preparar material y dedicar al menos una sesión a presentar las bases de la misma y ver como se traslada ese enfoque al trabajo que deben realizar.

Listado de entregables:

En todas las entregas debe indicarse el nombre completo de los estudiantes (nombrar a un coordinador del equipo):
a) Informe inicial
b) Presentación del informe inicial
c) Documentación de proyecto
d) Informe final del proyecto
e) Presentación del informe final
f) Ensayo final individual

g) Valoración del trabajo de los restantes miembros del equipo

h) Prueba integrada de conocimientos

i) La asistencia y participación

Recursos adicionales. Utilización de vídeos: la realización de entrevistas con miembros de la organización seleccionada y su grabación en soporte video gráfico se considera especialmente interesante. También podrán utilizarse vídeos para la presentación de los resultados del proyecto. El uso de vídeos incentiva a los estudiantes para aprender cómo diseñar y realizar entrevistas, cómo elaborar cuestionarios para la entrevista, cómo utilizar el vídeo como herramienta comunicativa, cómo grabar y editar los vídeos, etc. El uso de vídeos no implica que los equipos no deban recopilar y analizar otro tipo de información (documentos de la organización, encuestas, observación personal, etc.), siempre dentro de los límites del proyecto (no se debe recoger excesiva información).

Prioritariamente, los equipos entregarán sus documentos (textos, vídeos, etc.) a través de un portafolio (ej: google drive).

\section{RESULTADOS}

En el análisis de los resultados de la experiencia se han considerado aspectos tales como: resumen actividades estudiantes, rendimiento académico, valoración de la experiencia y principales ventajas encontradas.

\section{Resumen actividades estudiantes}

En la tabla 1 se recoge un resumen de las actividades y el tiempo (en horas), que los estudiantes han trabajado con ABP durante la asignatura.

Tabla 1.

Resumen actividades/horas del estudiante con ABP.

Actividades/Tiempo

Experiencia con metodología activa(Aprendizaje Basado en Problemas)
Totales

Parciales

$\begin{array}{ccccc}\text { Experiencia con metodología } & 1^{\mathrm{er}} & 2^{\circ} & 3^{\circ} & 4^{0} \\ \text { activa(Aprendizaje Basado en Problemas) } & \text { mes } & \text { mes } & \text { mes } & \text { mes }\end{array}$




\begin{tabular}{|c|c|c|c|c|c|}
\hline $\begin{array}{l}\text { adaptando su evaluación en estudios de } \\
\text { Organización de empresas }\end{array}$ & & & & & \\
\hline Lecturas y recensiones. & 5 & 5 & 5 & 5 & 20 \\
\hline $\begin{array}{l}\text { Preparación y/o informes de sesiones } \\
\text { prácticas. }\end{array}$ & 5 & 5 & 5 & 5 & 20 \\
\hline Trabajos e informes de temas. & 8 & 8 & 8 & 11 & 35 \\
\hline $\begin{array}{l}\text { Revisiones y reconducciones de los trabajos } \\
\text { (grupal y en tutoría). }\end{array}$ & 2 & 3 & 2 & 3 & 10 \\
\hline Estudiar para el examen & 5 & 5 & 5 & 5 & 20 \\
\hline Subtotales por meses & 25 & 25 & 25 & 29 & 105 \\
\hline
\end{tabular}

Rendimiento académico:

En la tabla 2 se presentan los resultados académicos globales de los estudiantes de la asignatura.

Tabla 2.

Resultados globales.

\begin{tabular}{ccc}
\hline Calificación & Porcentaje & $\mathbf{N}^{\mathbf{0}}$ estudiantes \\
\hline Matrícula Honor & & 1 \\
\hline Sobresaliente & $3 \%$ & 15 \\
\hline Notable & $52 \%$ & 10 \\
\hline Aprobado & $35 \%$ & 3 \\
\hline Suspenso & & 29 \\
\hline No presentado & $10 \%$ &
\end{tabular}

En general, se observa que ha sido positivo el desempeño de los estudiantes en cuanto a calificaciones se refiere. Además, de estos resultados, se han recogido los comentarios de la asignatura en la encuesta oficial del curso:

"Interesante/ Analizar el entorno de una empresa es apasionante/ Muy interesante, a la vez que educativa para conocer la economía en la actualidad/ Me gusta esta asignatura y los maestros, es útil para mi carrera en el futuro/ El profesor consigue involucrarte en la asignatura, muy productivas las clases./ El buen ambiente y la facilidad de aprendizaje y el entorno ameno además de las formas de trabajo posible que nos han facilitado/ Toca la vida real/ Dinámica de la clase/ La asignatura muy productiva, muy activa y bastante interesante me ha gustado y a la vez me ha sorprendido para bien/ Clase muy dinámicas".

\section{Valoración de la experiencia}

Los docentes han reconocido que en la experiencia se detectan las siguientes características:

a) Innovación (al desarrollar soluciones nuevas o creativas), porque permitió que los estudiantes se acercarán a la realidad a través de vías alternativas a la forma tradicional de la docencia.

b) Efectiva (cuando demuestra un impacto positivo y tangible de mejora), porque se logra un mayor compromiso de cada estudiante.

c) Sostenible (al mantenerse en el tiempo y producir efectos duraderos), porque los estudiantes pueden seguir tomando esa experiencia para 
desarrollar otro tipo de trabajos no solo académicos sino porque les hace dar cuenta de algunas de las necesidades que tienen las empresas en la actualidad y como buscar posibles soluciones.

d) Replicable (cuando es posible utilizarla como modelo para desarrollarla en otros contextos), porque esta metodología comenzó a desarrollarse en el área de la medicina y se ha extendido a múltiples disciplinas, con lo cual no hay inconveniente con que este tipo de experiencia se pueda extrapolar a otras asignaturas (siempre que se adapte al contexto o grupo donde se va a desarrollar).

La valoración del proceso de toda la experiencia es positiva, aunque reconozco que se tienen diferentes fases con cierto grado de complicación para el avance del empleo de esta metodología. En este caso vale la pena indicar que el contacto con la empresa es el momento más crítico del trabajo, debido a que no solo es la primera vez que lo estudiantes se enfrentan a presentar una solicitud de manera formal "profesional" a los responsables de las empresas, sino que es cuando se define si podrá contar con ese apoyo, brindando el acceso a la información que haga falta a los estudiantes. Las demás fases dependen en su mayoría de la orientación o reorientación que el profesor plantea a los estudiantes según van abordan los distintos aspectos del trabajo.

\section{Principales ventajas encontradas}

Esta experiencia proporciona a los estudiantes:

- Construir su proceso de aprendizaje dando la posibilidad de explorar conocimientos multidisciplinares.

- Practicar las habilidades organizativas y directivas.

- Integrar los conceptos teóricos de la asignatura y transformarlos en herramientas útiles para la solución de problemas en organizaciones reales.

- Propiciar la motivación y mayor compromiso en su aprendizaje.

- Desarrollar la capacidad para criticar y evaluar el trabajo propio y el ajeno.

- Fomentar el empleo de TIC.

- Avanzar en las competencias relativas al trabajo en equipo, la planificación y ejecución de trabajos, así como la ejecución de tareas con disponibilidad limitada de tiempo y de recursos.

En general, la metodología ABP fue una alternativa que se ajustó sin mayores inconvenientes a los requerimientos de la asignatura dado que los estudiantes pudieron conocer organizaciones reales, interactuar con las mismas, al analizar sus problemáticas tendiendo como referencia no solo el contenido de la asignatura sino de otras disciplinas, además del empleo de diferentes recursos de TIC. De esta manera, los estudiantes mejoraron en motivación y han podido desarrollar y ampliar sus conocimientos, habilidades y competencias.

\section{CONCLUSIONES}

La creación del Espacio Europeo de Educación Superior (EEES) que comenzó en la declaración de la Sorbona en 1998 y que se amplia y consolida con la declaración de Bolonia de 1999, ha abierto oportunidades para hacer modificaciones destacadas en educación. Algunos de los lineamientos del EEES, en concreto, implican renovaciones metodológicas y de evaluación del proceso enseñanza-aprendizaje.

En este marco, la experiencia educativa buscó contribuir al proceso de construcción del aprendizaje de los estudiantes a través del fomento de las metodologías activas (con el aprendizaje basado en problemas) y el empleo de las TIC en la asignatura de Organización de empresas. Este enfoque se planteó para propiciar que los alumnos fueran más participativos en su educación y ampliaran el empleo útil de recursos tecnológicos. De tal manera, que pudieran profundizar y asimilar mejor sus 
conocimientos, viéndose reflejado no solo en adquirir más competencias sino en las calificaciones de la asignatura.

Así, el fomento del aprendizaje de los estudiantes a través del fomento de una metodología alternativa (ABP) en la asignatura de Organización de empresas fue el objetivo de esta experiencia. Esta metodología fue punto de referencia, porque significó que los estudiantes pudieron acceder al conocimiento de organizaciones reales por otras vías. En cuanto a los informes y su respectiva presentación, los estudiantes han tenido que hacer un esfuerzo para trasladar sus conocimientos en una forma clara, concisa y dinámica, para mostrar la organización, el problema; contenidos de la asignatura; es decir, la teoría y la práctica. Estas estrategias resultaron provechosas para ordenar sus ideas y aclarar dudas de los contenidos y mejorar sus habilidades (pensamiento crítico y práctico, análisis y resolución de problemas, trabajo en equipo, entre otras). En este proceso fue útil el seguimiento y cambio en la forma de evaluar (con rúbricas, presentaciones, calificaciones).

Los cambios que se han planteado en la asignatura han obtenido en general una buena acogida y esto se corrobora no solo con la actitud de los estudiantes y su rendimiento académico sino con el mayor desarrollo de sus habilidades y competencias.

\section{REFERENCIAS}

Baysura, O. D., Altun, S., \& Toy, B. Y. (2016). Perceptions of teacher candidates regarding project-based learning. Eurasian Journal of Educational Research, 16(62), 15-36.

Bell, S. (2010). Project-based learning for the 21st century: Skills for the future. The Clearing House, 83(2), 39-43.

Cavus, N., Uzunboylu, H., \& Ibrahim, D. (2006). Combining collaborative learning with learning management systems in teaching programming language. En $2^{\circ}$ international open and distance learning symposium. Eskisehir, Turkey: Anadolu University.

Cavus, N., Uzunboylu, H., \& Ibrahim, D. (2007). Assessing the success rate of students using a learning management system together with a collaborative tool in web-based teaching of programming languages. Journal of educational computing research, 36(3), 301-321.

Declaración de Bolonia. (1999). El espacio europeo de educación superior. Declaración conjunta de los ministros europeos de educación, reunidos en Bolonia el, 19.

Exley, K., \& Dennick, R. (2007). Enseñanza en pequeños grupos en educación superior. Tutorías, seminarios y otros agrupamientos. Madrid: Narcea.

Fernández-Pérez, M. (1989). Así enseña nuestra Universidad. Hacia la construcción crítica de una didáctica universitaria. Madrid: UCM.

Goldstein, O. (2016). A project-based learning approach to teaching physics for preservice elementary school teacher education students. Cogent Education, 3(1), 1-12.

Jacques, S., Bissey, S., \& Martin, A. (2016). Multidisciplinary Project Based Learning Within a Collaborative Framework. International Journal of Emerging Technologies in Learning, 11(12), 36-44.

Lemus, L. (2006). Pedagogía: Temas fundamentales (5a. ed.). Buenos Aires: Kapelusz.

López-Noguero, F. (2007). Metodología participativa en la enseñanza universitaria. Madrid: Narcea. 
López-Pastor, V.M., Castejón, J., Sicilia-Camacho, A., Navarro-Adelantado, V., \& Webb, G. (2011). The process of creating a cross-university network for formative and shared assessment in higher education in Spain and its potential applications. Innovations in Education and Teaching International, 48(1), 79-90.

March, A. F. (2006). Metodologías activas para la formación de competencias. Educatio siglo $X X I, 24,35-56$.

Meyers, C., \& Jones, T. B. (1993). Promoting active learning: Strategies for the college classroom. San Francisco: Jossey-Bass.

Savery, J. R. (2015). Overview of problem-based learning: Definitions and distinctions. Essential readings in problem-based learning: Exploring and extending the legacy of Howard S. Barrows, 9, 5-15.

Zayapragassarazan, Z., \& Kumar, S. (2012). Active Learning Methods. Online Submission, 19(1), 3-5. 